\title{
Absolute and relative perceptual codes in young children
}

\author{
JIM DUFFY \\ Memorial University of Newfoundland, Corner Brook, Newfoundland, Canada \\ and
}

\author{
MARILYN T. ZIVIAN and ANN-MARIE DEBORAN \\ York University, Toronto, Ontario, Canada
}

\begin{abstract}
Three experiments were conducted to ascertain whether young children's perceptual codes, as evidenced by choice reaction times, were related to either the absolute difference or the ratio between stimuli. In Experiments 1 and 2, children were shown pairs of line lengths and were asked to choose either the longer or shorter line within each pair. In Experiment 3, children chose which of two birds on a map was closer to themselves or to the experimenter. In all three experiments, children's reaction times were related to both the absolute differences and ratios between pairs of stimuli. The findings suggest that perceptual judgments in young children may be based on both absolute and relational codes.
\end{abstract}

Human beings appear to undergo notable improvements in perceptual development between the ages of 5 and 10 years. Visual acuity, the speed of processing of visual information, and visual scanning strategies undergo rapid improvement during this period (Vurpillot, 1968; Williams, 1983). Given these developmental changes, one might question whether the perceptual codes of children contain the same type of information as those of adults. For instance, two effects in adults' relative judgments, the distance effect and the ratio effect, suggest that adults code both absolute differences and ratios when comparing stimuli; if children's judgments do not show either of these effects, there would seem to be a major difference between the perceptual codes of children and adults. However, if children also show these same effects, there would seem to be a degree of similarity between children's and adults' perceptual codes, despite the developmental differences in their perception.

\section{Distance Effect}

The term distance effect refers to a decrease in relative judgment time that occurs as the distance between stimuli

The present studies were conducted while Jim Duffy was a recipient of a Natural Science and Engineering Research Council Scholarship; they constituted part of his PhD dissertation. The authors wish to thank the students in a Psychology undergraduate class at York University for their help with the data collection in Experiments 2 and 3. Sol Minkin and John Fox helped with parts of the statistical analyses. Finally, the authors would like to thank the principals, teachers, and students of Arbor Glen and St. Sebastian schools for their cooperation. Preliminary results of Experiment 1 were presented at the University of Waterloo Conference on Child Development (May, 1982), and preliminary results of all three experiments were presented at the annual meeting of the Psychonomic Society (November, 1983). Reprints may be obtained from Jim Duffy at the Department of Psychology, Memorial University of Newfoundland, Sir Wilfred Grenfell College, Corner Brook, Newfoundland, $\mathrm{A} 2 \mathrm{H} 6 \mathrm{P9}$, Canada. along some dimension increases (Moyer \& Bayer, 1976). Thus, when judgments are made on the basis of a unidimensional attribute, reaction times (RTs) tend to be longer for pairs closer together on that attribute than for pairs farther apart on the attribute. Although the curve is usually linear, significant quadratic effects are sometimes found (Friedman, 1984). As will be seen below, the distance effect appears to be based on an absolute code that operates independently from the effect of a relative code based on stimulus ratios.

The absolute code that underlies the distance effect appears to be finely-grained, rather than coarse. In adults' judgments of numerous types of stimuli, subjective interval distances between stimuli have accounted for the distance effect better than have the ordinal distances between stimuli (Banks, White, Sturgill, \& Mermelstein, 1983, Experiments 2 and 3; Holyoak \& Walker, 1976; Kerst \& Howard, 1977).

The distance effect has been found for children from kindergarten age up to adulthood (Duncan \& McFarland, 1980; Sekuler \& Mierkiewicz, 1977). With digits used as stimuli, the distance effect has been found for both perceptual (same-different?) and memorial (which is longer?) tasks (Duncan \& McFarland, 1980). In addition to age differences for intercept values, the slope of the distance effect tends to decrease with age (Duncan \& McFarland, 1980; Sekuler \& Mierkiewicz, 1977). The intercept differences probably reflect differences in speed of processing, but the differences in slope may reflect either different distances on the representational continuum, or more noise in the representations of children than in those of adults (Sekuler \& Mierkiewicz, 1977).

\section{Ratio Effect}

Adult judgments of the lengths of lines and heights of triangles have yielded RTs that reflect both a typical dis- 
tance effect and an independent ratio effect (Munsterberg, 1894; Petrusic \& Jamieson, 1979). The term ratio effect refers to an increase in RT as the ratio of the stimuli approaches unity. There appears to be a lack of studies relating stimulus differences and ratios to children's choice RTs. In a length-discrimination experiment by Jones and Duffy (1982) designed to investigate speed-accuracy relations in preschoolers, some evidence was found for a ratio effect in children's RTs. However, in that study, ratios and differences were confounded.

The three experiments described below were designed to investigate whether there is a ratio effect, independent of an effect of absolute differences, in young children's judgments of perceptual stimuli. The first two studies are attempts to replicate the Jones and Duffy (1982) results for length discriminations.

\section{EXPERIMENT 1}

\section{Method}

\section{Subjects}

The children who participated in Experiment 1 were enrolled in the kindergarten classes of a public elementary school. Fifty subjects were chosen by means of a computer-generated random number table. Equal numbers of males and females were selected. Their mean age was 5 years and 2 months (range 4 years, 3 months, to 6 years, 3 months). The children were from a predominantly uppermiddle class background.

\section{Materials}

Pairs of horizontal lines of varying length were drawn in black ink on $20 \times 13 \mathrm{~cm}$ white index cards. The lines were $2 \mathrm{~mm}$ wide. Each pair was drawn in parallel, with $2.5 \mathrm{~cm}$ separating the lines. The top line was indented $3 \mathrm{~cm}$, the bottom line $4 \mathrm{~cm}$, from the left border of the card.

The lines varied in length by increments of $1.25 \mathrm{~cm}$ from 1.25 to $11.25 \mathrm{~cm}$, producing nine different line lengths: $1.25,2.50,3.75$, $5.00,6.25,7.50,8.75,10.00$, and $11.25 \mathrm{~cm}$. Pairs were made by taking all possible permutations of the lines, thus generating 72 pairs of lines: 36 with the longer line on the top and 36 with the longer line on the bottom. Lines of identical length were never paired.

The particular line lengths were chosen in order to vary ratios independently of absolute differences, although the design is not completely factorial. For example, pairs 1.25 and $2.50,2.50$ and $3.75,3.75$ and $5.00,5.00$ and $6.25,6.25$ and $7.50,7.50$ and 8.75, 8.75 and 10.00 , and 10.00 and 11.25 contain a constant absolute difference but varying ratios (from a ratio of .50 , for the pair 1.25 and 2.50 , to a ratio of .89 , for the pair 10.00 and 11.25 ). Likewise, there are pairs with a constant ratio but with varying absolute differences (e.g., pairs 1.25 and 2.50, 2.50 and 5.00, 3.75 and 7.50 , and 5.00 and 10.00 ).

In addition to the 72 pairs of lines, four practice cards containing lines 1.25 and $12.50 \mathrm{~cm}$ in length were used. On two of the practice cards, the longer line was on the top, and on the other two, the longer line was on the bottom.

The children's response times were measured by means of a handheld Cronus digital stopwatch. The experimenter was practiced in the use of the stopwatch, so that the measured time difference in the starting and stopping of the watch between trials was usually within a few hundredths of a second. The use of a hand-held stopwatch has previously proved to be sensitive to psychophysical judgments in young children (cf. Jones \& Duffy, 1982).

\section{Procedure}

Each child was tested individually. The children were told that they would be shown pictures of lines and that they were to point to the longer line. The children were instructed to respond as quickly and as accurately as they could. If a child's hands were not spontaneously placed on the table at which he or she was seated, the child was asked to place them there.

Each child was first shown the four practice cards, followed immediately by the 72 experimental pairs. Each child saw a unique random order of the experimental pairs.

The experimenter turned over each card with one hand while starting the stopwatch with the other hand. When the child's finger came to rest on the top of either line, the latency and accuracy of the response were recorded by the experimenter. The children were told if they had made an error. Trials that resulted in errors were repeated after all of the other trials had been completed. The testing took approximately $20 \mathrm{~min}$ for each child.

\section{Results}

\section{Errors}

No child made an error on any of the practice trials. Practice trials were excluded from any further analyses. On the experimental trials, errors occurred less than $2 \%$ of the time. In all of the following analyses, only RTs for correct choices were used. Whenever an error occurred, the RT for the repeated trial was employed in the analysis.

\section{General Linear Model}

A general linear model (see Table 1), similar to a multiple regression model, was tested against the RT data. The model contained the following variables: individual differences in children's responding, differences between the line lengths, ratios between the line lengths, and interactions. In addition, the model was composed of three levels of complexity: linear, quadratic, and cubic functions. At each level of complexity, each variable was treated as if it had been entered into the equation last, partialing out the common variance and thus statistically controlling for the effect of one variable on another. Table 1 shows the model with tests of each variable. Overall, $r^{2}=.44$.

Individual differences. The results in Table 1 show some evidence of individual differences (ID) in the functions relating differences and ratios to RT. Some interactions containing ID reached significance at the .05 level. This evidence is somewhat weak, however, due to the large number of tests on the same data. A more conservative significance level of .003 (the alpha level, .05, divided by the number of variables, 19 ; see Cohen $\&$ Cohen, 1983) yielded the result that none of the interactions containing ID were significant.

Differences. As can be seen from Figure 1, RTs generally decreased as the differences in length between the lines in a pair increased.

Ratios. Figure 2 presents the median choice RTs for the ratio of the shorter to the longer line in a pair. There was a nonlinear increase in RT as the ratios increased. 
Table 1

General Linear Models: Experiments 1 and 2

\begin{tabular}{|c|c|c|c|c|}
\hline \multirow[b]{3}{*}{ Variable } & \multicolumn{4}{|c|}{ Experiment } \\
\hline & \multicolumn{2}{|c|}{$\begin{array}{c}\text { I } \\
\text { Longer? }\end{array}$} & \multicolumn{2}{|c|}{$\begin{array}{c}2 \\
\text { Smaller? }\end{array}$} \\
\hline & $d f$ & $F$ & $d f$ & $F$ \\
\hline General Model & 499 & $4.81 \S$ & 639 & 4.608 \\
\hline Error & 3,100 & & 3,939 & \\
\hline Individual differences (ID) & 49 & 1.35 & 63 & 1.21 \\
\hline Differences & 1 & $17.53 \S$ & 1 & $5.78^{*}$ \\
\hline Differences $\times I D$ & 49 & 1.25 & 63 & 1.15 \\
\hline Ratios & 1 & 26.878 & 1 & 15.428 \\
\hline Ratios $\times$ ID & 49 & $1.45^{*}$ & 63 & 1.30 \\
\hline Differences $^{2}$ & 1 & 14.618 & 1 & 3.49 \\
\hline Differences $^{2} \times$ ID & 49 & 1.15 & 63 & 1.10 \\
\hline Ratios $^{2}$ & 1 & $30.36 \S$ & 1 & 22.378 \\
\hline Ratios $^{2} \times$ ID & 49 & $1.51 *$ & 63 & $1.42 *$ \\
\hline Differences $\times$ ratios & 1 & 22.128 & 1 & $10.20 \ddagger$ \\
\hline Differences $\times$ ratios $\times$ ID & 49 & 1.32 & 63 & 1.21 \\
\hline Differences $^{3}$ & 1 & $12.32 \S$ & 1 & 2.15 \\
\hline Differences $^{3} \times$ ID & 49 & 1.08 & 63 & 1.03 \\
\hline Ratios $^{3}$ & 1 & 34.618 & 1 & 31.948 \\
\hline Ratios $^{3}$ and ID & 49 & $1.57 t$ & 63 & $1.56+$ \\
\hline Differences $^{2} \times$ ratios & 1 & $18.95 \S$ & 1 & $6.33^{*}$ \\
\hline Differences $^{2} \times$ ratios $\times$ ID & 49 & 1.21 & 63 & 1.16 \\
\hline Differences $\times$ ratios $^{2}$ & 1 & 24.028 & 1 & $15.73 \S$ \\
\hline Differences $\times$ ratios $\times$ ID & 49 & 1.37 & 63 & 1.29 \\
\hline
\end{tabular}

As can be seen from Figure 2, this increase appears predominately for ratios above 0.6 .

Ratios with differences between the line lengths held constant. Figure 3 presents median RTs for three levels of difference between the line lengths: $1.25,2.50$, and $3.75 \mathrm{~cm}$. In general, the RTs decrease with each increasing level of difference. In addition, at each level of difference depicted, there is a cubic relationship between RTs and ratios. The pairs of lines that yield smaller ratios tend to contain smaller lines, and the median RTs for responses to these are shown in the first and second points from the left on each curve in Figure 3. The significant cubic function between ratios and RT thus appears to be due to the children's having taken relatively longer for pairs that contained only short lines than for pairs that contained medium-length or long lines, in what might otherwise be approximately monotonically increasing curves.

For the first and second points from the left on each difference curve (see Figure 3), the children's RTs increase as the ratios between the lines increase. Thus, the

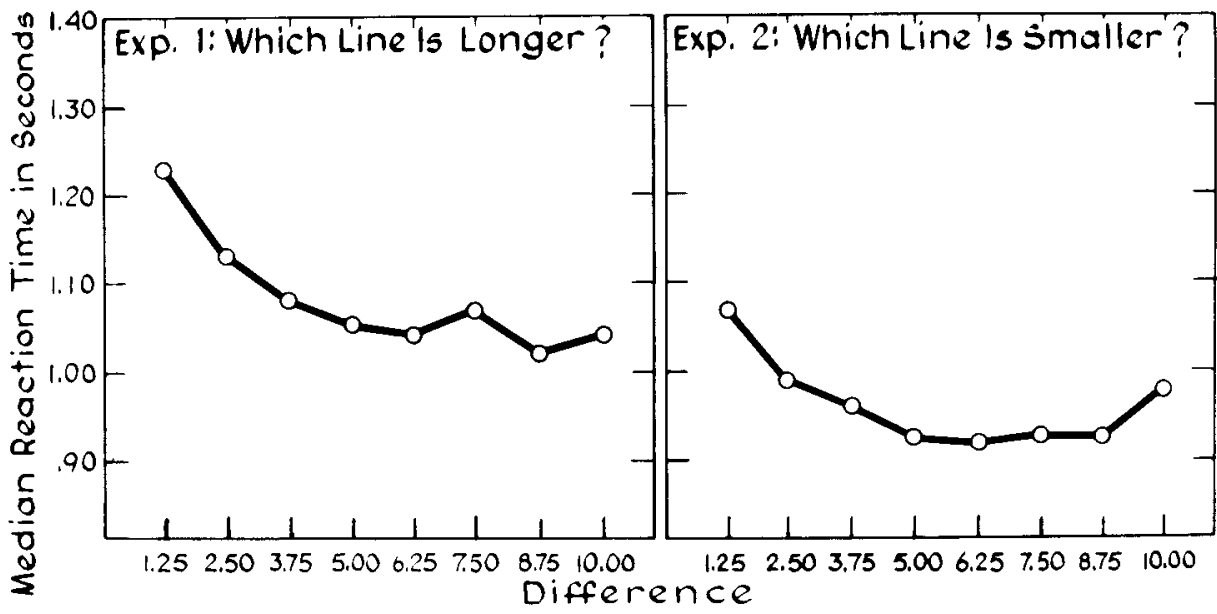

Figure 1. Median RTs for the absolute differences between pairs of stimuli. In the left-hand panel are the RTs obtained for subjects choosing the longer line length (Experiment 1); in the right-hand panel, RTs for subjects choosing the smaller line length (Experiment 2). 


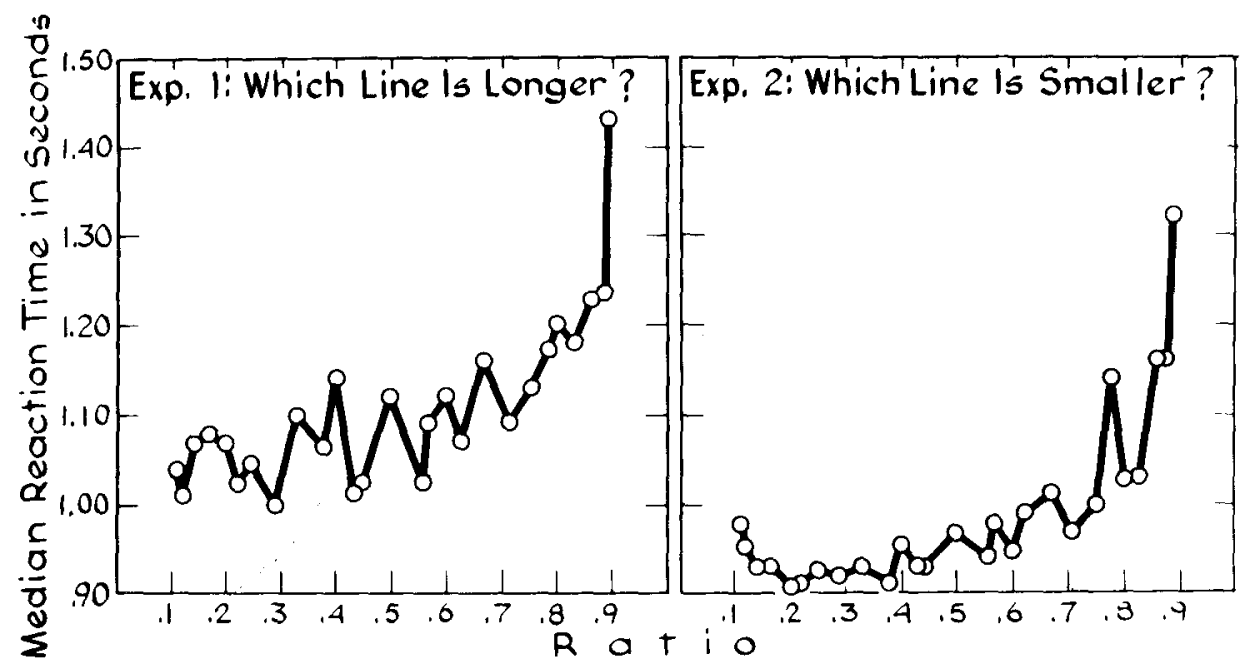

Figure 2. Median RTs for the ratios between pairs of stimuli. In the left-hand panel are the RTs obtained for subjects choosing the longer line length (Experiment 1); in the right-hand panel, RTs for subjects choosing the smaller line length (Experiment 2).

data depicted in Figure 3 suggest that a ratio effect may be present for ratios below 0.6 .

\section{Discussion}

The results of the analyses above indicate that the preschool children's choice RTs were influenced by both the absolute difference and the ratio between the stimuli. Experimental and statistical controls demonstrate an effect of ratios independent of differences, and vice versa. The present findings are stronger than earlier tentative findings presented by Jones and Duffy (1982) regarding the processing of differences and ratios by young children. Since these effects have also been found in the adult literature, the results suggest a close correspondence between the perceptual codes of young children and adults.

A separate effect that may be present in the data is the semantic congruity effect. This is an interaction between instructions and stimulus magnitude (cf. Banks, 1977; Moyer \& Dumais, 1978). In the present study, RTs were relatively greater for choosing between the larger of two small stimuli than they were for choosing the larger of two large stimuli. This resulted in the cubic function relating RTs to ratios.

\section{EXPERIMENT 2}

Our purpose in Experiment 2 was to replicate the findings of the processing of ratios and differences by young children. In the present experiment, the method in Experiment 1 was extended to include a different form of the comparative (smaller rather than longer) and a different sample of children.

\section{Method}

\section{Subjects}

Sixty-four kindergarten children were tested. The children in Experiment 2 came from another school than did the children in Experiment 1 , and they were mainly from lower middle class, Italian and Portuguese immigrant families. For most of these children, English was the second language. Their ages varied from 4 years, 6 months, to 6 years, 2 months. The mean age was 5 years. There were equal numbers of males and females.

\section{Materials and Procedure}

The materials were the same as those used in Experiment 1. The procedure differed from that in Experiment 1 in the following ways: Each child was instructed to point to the smaller line of the pair. On the first few practice trials, it was found that many of the children did not understand the word shorter in the present context,

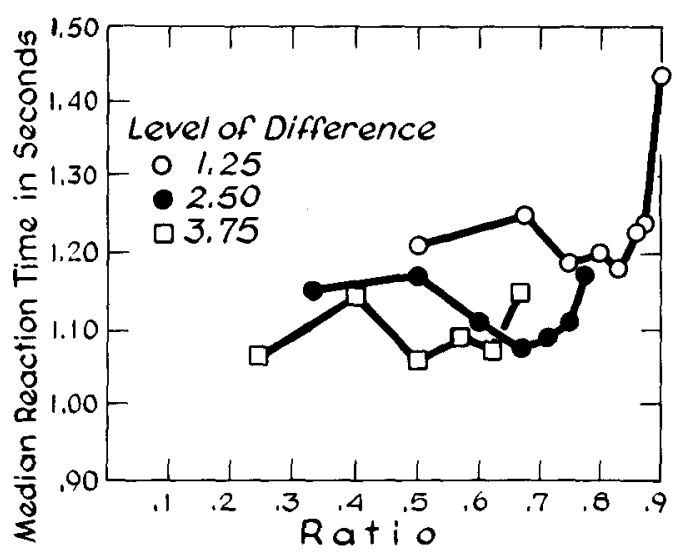

Figure 3. Median RTs for the ratios between the line lengths at three levels of length difference (separate symbols), for subjects choosing the longer of two lines in a pair (Experiment 1 ). 
but that they did understand the word smaller (see Clark \& Clark, 1977). The procedure was then changed, and the children were asked to point to the smaller line. One experimenter controlled the presentation of the stimuli, while another operated the stopwatch and recorded the accuracy and latency of all children.

\section{Results}

\section{Errors}

It was common for the children to make errors on the first few practice trials, but, with feedback, by the beginning of the experimental trials they pointed correctly to the smaller of the two lines. Errors occurred less than $3 \%$ of the time on experimental trials. Only RTs for correct choices were used in the following analyses.

\section{General Linear Model}

The general linear model used in Experiment 1 was tested against the data from Experiment 2. Table 1 shows the model with tests of each variable. Overall, $r^{2}=.43$.

Individual differences. At an alpha level of $.003(.05$ divided by the number of variables, 19) none of the interactions containing ID were significant. However, ratios squared by ID and ratios cubed by ID were significant at the .05 level in both Experiment 1 and Experiment 2. These findings suggest a possibility of individual differences in the shape of the ratio functions, the exact nature of which is unclear.

Differences. As in Experiment 1, RTs tended to decrease as the differences in length between the lines in a pair increased (see Figure 1). The linear component for differences showed significance only at the .05 level. The squared and cubic functions for differences, by themselves, were not significant.

Ratios. Figure 2 presents the median choice RTs for the ratio of the shorter to the longer line in a pair. There was a nonlinear increase in RT as the ratios increased, as was the case in Experiment 1.

Ratios and differences. There were significant interactions between ratios and differences, differences squared

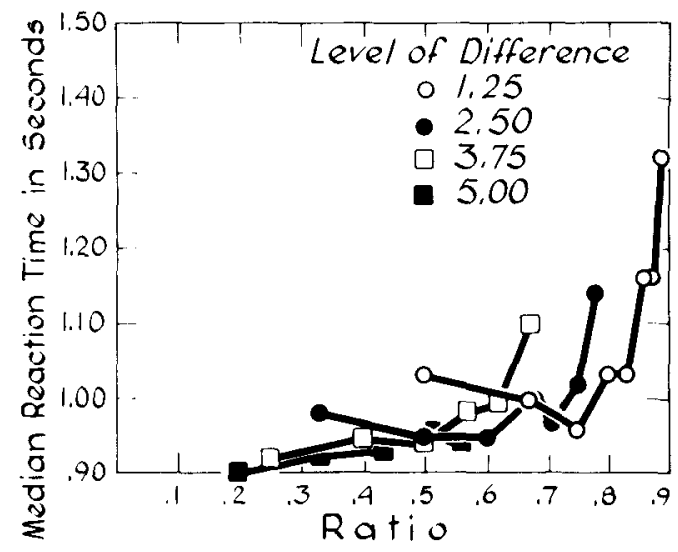

Figure 4. Median RTs for the ratios between the line lengths and for four levels of length difference (separate symbols), for subjects choosing the smaller of two lines in a pair (Experiment 2).

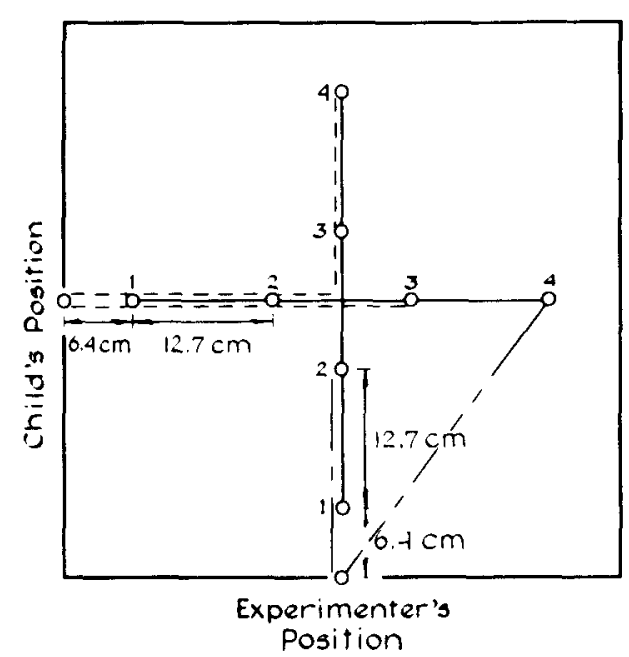

Figure 5. Spatial configuration for subjects choosing the closer of two objects (Experiment 3).

by ratios, and differences by ratios squared. Figure 4 displays median RTs for ratios at four different levels of differences. The ratio functions overlap at the various levels of differences. At the 1.25- and 2.50-cm levels of difference, RTs first decrease and then increase with ratios. For differences of 3.75 and $5.00 \mathrm{~cm}$, the ratio functions are closer to linear.

\section{Discussion}

The findings in Experiment 2 replicate those in Experiment 1 . Ratios and differences were both related to young children's choice RTs. Thus, ratio and difference effects have been found with two types of instructions, and with two very different samples: children from upper middle class, English-speaking families and children from working class, non-English-speaking, immigrant families.

\section{EXPERIMENT 3}

Experiment 3 was an attempt to extend the finding of children's processing of differences and ratios of length to judgments of distance.

\section{Method}

\section{Subjects}

Forty children from kindergarten and Grade 1 were tested. The children were from the same school and had the same general background as those in Experiment 2. Their mean age was 6 years, 2 months (range 5 years, 2 months, to 7 years, 8 months).

\section{Materials}

The stimuli consisted of two small cardboard birds, one red and one blue, and a cardboard map. The birds were placed at various points along the cardboard map. The cardboard map was $50.9 \mathrm{~cm}$ square (see Figure 5). Two intersecting lines were drawn on the cardboard; these will be referred to as an $x$-axis and a $y$-axis. There were four points on each axis, each point being denoted by a very faint line. Each axis started $6.4 \mathrm{~cm}$ from the board's edge. There was a distance of $12.7 \mathrm{~cm}$ between each adjacent point on either axis. 
The children's RTs were recorded by means of a hand-held Cronus digital stopwatch.

\section{Procedure}

Each child was tested individually. The experimenter and the child sat at a table on which the map had been placed. The child sat so that his or her head was directly above the edge of the map nearest to position 1 on the $x$-axis (see Figure 5). The experimenter sat so that her head was directly above the edge of the map nearest to position 1 on the $y$-axis. An adult who timed the responses sat opposite the experimenter.

Each child was given two pretests in which the child was asked to say the color of the bird that was closest to him or to her and also the color of the bird that was closest to the experimenter. Each child was then given 32 test trials. On every trial, one bird was always placed on the $x$-axis, while the other was always placed on the $y$-axis. Within this constraint, the positioning of the birds for each trial was randomly determined across subjects. Prior to a test trial, an opaque screen was placed between the child and the map, so that the child could not see the configuration until the screen was lifted. In one set of 16 trials, the children were asked which of the birds was closest to them; in the other set, they were asked which of the birds was closest to the experimenter (the order of the two sets of questions was counterbalanced across subjects). The children answered by saying "red," "blue," or "neither." The color of the bird on each axis was counterbalanced. RTs were measured from the time at which the screen was removed to the time at which the child gave a response. Feedback was given about the accuracy of each response.

\section{Results}

\section{Independent variables}

Eight measurements taken for each stimulus pair were compared with the RTs. These measurements resulted from combining: (1) either ratios or differences, (2) either measurements taken from where the child was positioned relative to the stimuli or measurements taken from where the experimenter was positioned relative to the same stimuli, and (3) either a Euclidian or a city-block metric. Examples of a Euclidian and a city-block measurement to a stimulus pair are shown in Figure 5. From the experimenter's position, the two lines with just a few breaks in them depict a Euclidian metric. From the child's position, the two broken lines depict a city-block metric. Cityblock metrics permit only horizontal and vertical measurements between two points; diagonal measurements were not permitted. There is evidence, at least for adults, that subjects sometimes use a metric similar to the cityblock metric when making judgments between stimuli that differ on two perceptually distinct dimensions (Attneave, 1950; Carroll \& Wish, 1974; Shepard, 1964).

\section{Multiple Regression Analyses on RTs}

Separate multiple regression analyses were conducted for the questions "closer to child?" and "closer to experimenter?" Only RTs for correct responses (90\% of total responses) were included. RTs of $6 \mathrm{sec}$ or more were excluded from the analyses. The regression models contained a forced ordering of the eight types of measurement. The order of the variables was the same for both analyses. From first to last variable, the order was as fol- lows: (1) Euclidian differences measured from the child's perspective, (2) Euclidian ratios from the child's perspective, (3) Euclidian differences from the experimenter's perspective, (4) Euclidian ratios from the experimenter's perspective, (5) city-block differences from the child's perspective, (6) city-block ratios from the child's perspective, (7) city-block differences from the experimenter's perspective, and (8) city-block ratios from the experimenter's perspective. It should be noted that because individual differences and interactions were not entered into the regression equations, one would expect the values of $r^{2}$ to be lower in Experiment 3 than it was in the two previous experiments.

"Closer to child?" Overall, for the entire model, $F(8,574)=9.74, p<.0001, r^{2}=.12$. When the children were asked the question of which was "closer to you," their RTs were significantly related to only two measurements: Euclidian differences from the child's perspective $[F(1,574)=58.99, p<.0001]$, and Euclidian ratios from the child's perspective $[F(1,574)=13.36$, $p<.0005]$. RTs tended to decrease as Euclidian differences increased and to increase as Euclidian ratios (of the shorter to the longer distance) increased (see Table 2).

"Closer to experimenter?" Overall, for the entire model, $F(8,555)=5.90, p<.0001, r^{2}=.08$. When the children were asked the question of which was "closer to the experimenter," their RTs were significantly related to three measurements: Euclidian differences from the experimenter's perspective $[F(1,555)=26.65, p<.0001]$, Euclidian ratios from the experimenter's perspective $[F(1,555)=9.03, p<.005]$, and city-block ratios from the experimenter's perspective $[F(1,555)=4.31, p<$ .05]. RTs tended to: decrease as Euclidian differences (from the experimenter's perspective) increased, to increase as Euclidian ratios (from the experimenter's perspective) increased, and to increase as city-block ratios (from the experimenter's perspective) increased (see Table 2).

\section{Errors}

A total of 122 errors $(10 \%$ of the total number of responses) were made. There was no significant difference in the number of errors between the "closer to child" and "closer to experimenter" questions $\left[\chi^{2}(1, N=122)=\right.$ $2.32, p>.1]$.

Given the relatively high proportion of errors, an attempt was made to understand the reason for some of the errors. Sixty of the 122 errors occurred when a child's decision would be quite difficult (ratios varying from .98 to 1.00 and differences ranging from 0 to $.5 \mathrm{~cm}$ ) if it were based on one type of measurement (e.g., Euclidian distance from the child's perspective), while a decision based on any of the other three types of measurement (e.g., Euclidian distance from the experimenter's perspective, or city-block distance from either the child's or the experimenter's perspective) would be comparatively easy (ratios varying from .14 to .84 and differences ranging from 5.1 to $38.1 \mathrm{~cm}$ ). The 60 errors that occurred under the above conditions were classified by question and type 
Table 2

Median Reaction Times for the Significantly Related Types of Measurement in Experiment 3 (All Subjects Included)

\begin{tabular}{|c|c|c|c|c|c|c|c|c|}
\hline \multirow[t]{3}{*}{ Question } & \multicolumn{8}{|c|}{ Type of Measurement } \\
\hline & \multicolumn{8}{|c|}{ Euclidean Difference } \\
\hline & 0.5 & 5.1 & 7.3 & 12.5 & 12.9 & 18.1 & 20.0 & 25.6 \\
\hline Closer to the child?* & 1.67 & 1.65 & 1.34 & 1.60 & 1.29 & 1.35 & 1.12 & 1.16 \\
\hline \multirow[t]{3}{*}{ Closer to the experimenter? +} & 1.70 & 1.83 & 1.61 & 1.69 & 1.54 & 1.60 & 1.35 & 1.40 \\
\hline & \multicolumn{8}{|c|}{ Euclidean Ratio } \\
\hline & 0.20 & 0.24 & 0.59 & 0.72 & 0.84 & 0.98 & & \\
\hline Closer to the child?* & 1.27 & 1.36 & 1.45 & 1.56 & 1.67 & 1.59 & & \\
\hline \multirow[t]{3}{*}{ Closer to the experimenter? $\dagger$} & 1.40 & 1.35 & 1.54 & 1.64 & 1.84 & 1.70 & & \\
\hline & \multicolumn{8}{|c|}{ City-Block Ratio } \\
\hline & 0.14 & 0.20 & 0.43 & 0.60 & 0.71 & 0.99 & 1.00 & \\
\hline Closer to the experimenter? $†$ & 1.40 & 1.54 & 1.35 & 1.61 & 1.68 & 1.83 & 1.69 & \\
\hline
\end{tabular}

*Measured from the child's perspective. †Measured from the experimenter's perspective.

Table 3

Frequency of Error $\times$ Type of Measurement and Question

\begin{tabular}{lcc}
\hline & \multicolumn{2}{c}{ Question } \\
\cline { 2 - 3 } \multicolumn{1}{c}{ Type of Measurement } & "Which is closer to & "Which is closer to \\
you (child)?" & me (experimenter)?" \\
\hline Euclidean (experimenter's perspective) & 19 & 4 \\
City-block (experimenter's perspective) & 5 & 2 \\
Euclidean (child's perspective) & 1 & 16 \\
City-block (child's perspective) & 2 & 11 \\
\hline
\end{tabular}

of measurement (see Table 3). These 60 errors are significantly related to measurement set and question $\left[\chi^{2}(3, N=60)=32.77, p<.001\right]$. It seems that the children may sometimes have used the Euclidian distance from the experimenter when answering the question "which is closer to you (the child)?" It also appears that the children may sometimes have used Euclidian and cityblock distances from themselves when asked "which is closer to me (the experimenter)?" In these cases, the measures were often independent of the correct answer. Thus, it is not surprising that children made mistakes in these instances.

\section{Discussion}

It was found that young children's processing of ratios and differences did extend to the task of choosing the closer of two objects. The place from which the distances were processed by the children (as evidenced by the RTs) depended on the question that was asked of them. The children processed the ratios and differences from the birds to the experimenter when they were asked which of the birds was closer to the experimenter. The children processed the distances from the birds to themselves when they were asked which of the birds was closer to them.

The RT data for correct responses, and an analysis of some of the errors, suggest not only that Euclidian distances are being processed, but also that city-block distances are sometimes being processed by the children as well. It is not clear whether the processing of city-block distances is specific to the display used in the present experiment (which is clearly conducive to processing cityblock distances), or whether it represents a more general processing mode.

\section{GENERAL DISCUSSION}

Unique effects of ratios and differences on children's choice RTs have been demonstrated in each of the three experiments in the present paper. These effects were robust enough to occur despite the differences in instructions, stimuli, and subject populations in the various experiments. These results are unlikely to be an artifact of the experimenter's use of hand-held stopwatches to measure RT. Although the experimenters in Experiments 1 and 3 were conscious of the hypotheses, the experimenters in Experiment 2 were not aware of the expected results. In addition, in a recent memory experiment (Duffy, 1990) in which a computer was used to display stimuli and to record RTs, an effect of ratios and differences on children's choice RTs was also found. Thus, it is unlikely that the present results were due to unintentional experimenter bias.

The results of the experiments in the present study indicate that children do not code stimuli in a coarse manner, such as might be described by an ordinal scale, but instead code stimuli in enough detail to be described in 
terms of a ratio scale. However, it may be that information about length, or distance, is not represented along a single internal dimension. The ratio and difference information could be stored separately, as in a superpositional model. In this type of a model, pieces of information about an object or concept are stored at separate nodes, and the relationships between the pieces of information are represented by the connections between the nodes (Oden, 1987). In the case of the stimuli in the present experiment, the information about ratios and differences would be stored at separate locations rather than embedded in an internally constructed ratio scale dimension. The significant quadratic and cubic trends found in the present set of experiments for ratios, but not for differences, may be evidence that information about ratios and differences is stored separately. If both ratios and differences were stored along the same dimension, then one would expect any nonlinear trends to show up for both ratios and differences.

Despite the fact that the perceptual abilities of young children are not equivalent to those of adults, the perceptual codes used by young children appear to be quite like those of adults. Adults code both the absolute difference and the ratio of pairs of line lengths (Munsterberg, 1894), and Experiments 1 and 2 demonstrated that young children do so as well. In addition, adults sometimes process a city-block metric (Attneave, 1950; Carroll \& Wish, 1974; Shepard, 1964), as did the children in Experiment 3.

It may be that the RT effects found for ratios and differences between stimuli reflect some kind of basic processing by the visual system of these physical aspects of objects. This appears to be a type of processing that does not change radically from early childhood to adulthood.

\section{REFERENCES}

Attneave, F. (1950). Dimensions of similarity. American Journal of Psychology, 63, 516-556.

BANKs, W. P. (1977). Encoding and processing of symbolic information in comparative judgements. In G. H. Bower (Ed.), The psychology of learning and motivation (Vol. 11, pp. 101-159). New York: Academic Press.

Banks, W. P., White, H., Sturgill, W., Mermelstein, R. (1983). Semantic congruity and expectancy in symbolic judgments. Journal of Experimental Psychology: Human Perception \& Performance, 9, $560-582$.

Carroll, J. D., Wish, M. (1974). Multidimensional perceptual models and measurement methods. In E. C. Carterette \& M. D. Friedman (Eds.), Handbook of perception (Vol. 2, pp. 391-447). New York: Academic Press.

Clark, H. H., Clark, E. V. (1977). Psychology and language: An introduction to psycholinguistics. New York: Harcourt Brace Jovanovich.

CoHEN, J., \& CoHEN, P. (1983). Applied multiple regression/correlation analysis for the behavioral sciences. Hillsdale, NJ: Erlbaum.

DUFFY, J. (1990). Children's and adult's memorial representations of length. Unpublished manuscript

Duncan, E. M., \& MCFarland, C. E., JR. (1980). Isolating the effects of symbolic distance and semantic congruity in comparative judgments: An additive-factors analysis. Memory \& Cognition, 8, 612-622.

Friedman, W. J. (1984). Analog and semantic models of judgments about the months of the year. Memory \& Cognition, 12, 306-313.

HolyoAk, K. J., W Waker, J. H. (1976). Subjective magnitude information in semantic orderings. Journal of Verbal Learning \& Verbal Behavior, 15, 287-299.

JONES, B., \& DUFFY, J. (1982). An analysis of performance by preschool children on the KRISP and on a length discrimination task. Acta Psychologica, 52, 197-211.

Kerst, S. M., \& Howard, J. H., JR. (1977). Mental comparisons for ordered information on abstract and concrete dimensions. Memory \& Cognition, 5, 227-234.

Moyer, R. S., \& BAYER, R. H. (1976). Mental comparison and the symbolic distance effect. Cognitive Psychology, 8, 228-246.

MoYer, R. S., \& Dumais, S. T. (1978). Mental comparison. In G. H. Bower (Ed.), The psychology of learning and motivation (Vol. 12, pp. 117-155). New York: Academic Press.

Munsterberg, H. (1894). Studies from the Harvard psychological laboratory (I).C. A psychometric investigation of the psycho-physic law. Psychological Review, 1, 45-51.

ODEN, G. C. (1987). Concept, knowledge, and thought. Annual Review of Psychology, 38, 203-227.

Petrusic, W. M., \& Jamieson, D. G. (1979). Resolution time and the coding of arithmetic relations on supraliminally different visual extents. Journal of Mathematical Psychology, 19, 89-107.

Sekuler, R., \& Mierkiewicz, D. (1977). Children's judgments of numerical inequality. Child Development, 48, 630-633.

SHEPARD, R. N. (1964). Attention and the metric structure of the stimulus space. Journal of Mathematical Psychology, 1, 54-87.

VURPILLOT, E. (1968). The development of scanning strategies and their relation to visual differentiation. Journal of Experimental Child Psychology, 6, 632-650.

Williams, H. G. (1983). Perceptual and motor development. Englewood Cliffs, NJ: Prentice-Hall.

(Manuscript received November 22, 1988; revision accepted for publication January 9, 1990.) 